\title{
Gigaton Volume Detector in Lake Baikal: status of the project
}

A.D. Avrorin ${ }^{a}$, A.V. Avrorin ${ }^{a}$, V.M. Aynutdinov ${ }^{a}$, R. Bannash ${ }^{g}$, I.A. Belolaptikov" V.B. Brudanin ${ }^{b}$, N.M. Budnev ${ }^{c}$, I.A. Danilchenko ${ }^{a}$, G.V. Domogatsky ${ }^{a 1}$, A.A.Doroshenko ${ }^{a}$, R. Dvornickýb,h A.N. Dyachok ${ }^{c}$, Zh.-A.M. Dzhilkibaev' ${ }^{a}$, L. Fajt $^{b, h, i}$, S.V. Fialkovsky ${ }^{e}$, A.R. Gafarov ${ }^{c}$, K.V. Golubkov ${ }^{a}$, T.I. Gress ${ }^{c}$, Z. Hons ${ }^{b}$, K.G.Kebkal ${ }^{g}$, O.G. Kebkal ${ }^{g}$, M.M. Kolbin ${ }^{b}$, K.V. Konischev ${ }^{b}$, A.V. Korobchenko ${ }^{b}$, A.P.Koshechkin ${ }^{a}$, F.K. Koshela, A.V. Kozhin ${ }^{d}$, V.F. Kulepov, D.A. Kuleshov', M.B.Milenin ${ }^{e}$, R.R.Mirgazov ${ }^{c}$, E.R. Osipova ${ }^{d}$, A.I. Panfilov ${ }^{a}$, L.V. Pan'kov ${ }^{c}$, D.P.Petukhov ${ }^{a}$, E.N.Pliskovsky ${ }^{b}$, M.I., Rozanov ${ }^{f}$, E.V. Rjabov ${ }^{c}$, G.B.Safronov ${ }^{b}$, B.A.Shaybonov $^{b}$, M.D.Shelepov ${ }^{a}$, F. Simkovic ${ }^{b, h, i}$, A.V. Skurikhin ${ }^{d}$, I.Stekl ${ }^{i}$, O.V. Suvorova ${ }^{a}$, V.A., Tabolenkoc, B.A.Tarashansky ${ }^{c}$, S.A.Yakovlev ${ }^{g}$, A.V.Zagorodnikov ${ }^{c}$ and V.L. Zurbanov ${ }^{c}$

\author{
${ }^{a}$ Institute for Nuclear Research, Moscow, 117312 Russia \\ ${ }^{b}$ Joint Institute for Nuclear Research, Dubna, 141980 Russia \\ 'Irkutsk State University, Irkutsk, 664003 Russia \\ ${ }^{d}$ Institute of Nuclear Physics, Moscow State University, Moscow, 119991 Russia \\ ${ }^{e}$ Nizhni Novgorod State Technical University, Nizhni Novgorod, 603950 Russia \\ ${ }^{f}$ St. Petersburg State Marine Technical University, St. Petersburg, 190008 Russia \\ ${ }^{g}$ EvoLogics, Germany \\ ${ }^{h}$ Comenius University, Bratislava, Slovakia \\ ${ }^{i}$ Czech Technical University in Prague, Prague, Czech Republic
}

\begin{abstract}
Baikal-GVD is a kilometer-scale neutrino telescope under construction in Lake Baikal, which will be formed by multi-megaton subarrays - clusters of strings. A first demonstration cluster "Dubna" has been deployed in 2015 and comprises 192 optical modules (OMs). In 2016 this cluster was upgraded to the baseline configuration which comprises $288 \mathrm{OMs}$ arranged at eight strings. The second full-scale GVD-cluster was deployed and put in operation in 2017. We review the present activity towards the GVD implementation and discuss some selected results obtained with the "Dubna" cluster.
\end{abstract}

XVII International Workshop on Neutrino Telescopes 13-17 March 2017

Venezia, Ital

\footnotetext{
${ }^{1}$ Speaker domogats@yandex.ru
} 


\section{Introduction}

The construction of the Baikal-GVD neutrino telescope [1] is motivated by its discovery potential in astrophysics, cosmology and particle physics. Its primary goal is the detailed study the diffuse flux of high-energy cosmic neutrinos and the search for their sources. It will also search for dark matter candidates (WIMPs), for neutrinos from the decay of super heavy particles, for magnetic monopoles and other exotic particles. It will also be a platform for environmental studies in Lake Baikal. The site chosen for the experiment is in the southern basin of Lake Baikal. Here, the combination of hydrological, hydro-physical, and landscape factors is optimal for deployment and operation of the neutrino telescope. The water depth is about $1360 \mathrm{~m}$ at distances beginning from about three kilometers from the shore. The water transparency is characterized by an absorption length of about $20-25 \mathrm{~m}$ and a scattering length of $30-50 \mathrm{~m}$. The water luminescence is moderate at the detector site.

Baikal-GVD is rooted in the long-term operation of its predecessor NT200 [2]. During the Design Study (2008-2010) [3] the basic elements of GVD - new optical modules (OMs), FADC readout units, underwater communications and trigger systems - have been developed, produced and tested in-situ. During the Preparatory Phase (2011-2015) [4], comprehensive in-situ tests of all elements and systems of the future detector as part of several engineering arrays have been performed. The Preparation phase was concluded in 2015 with the deployment of a demonstration cluster comprising 192 OMs. The construction of the first phase of Baikal-GVD (GVD-I) was started in 2016 by deployment of the first cluster in its baseline configuration, consisting of 288 OMs arranged at eight $525 \mathrm{~m}$ long strings. In 2017, the array was upgraded by the deployment of the second GVD cluster. Commissioning of GVD-I ( 8 clusters, volume $0.4 \mathrm{~km}^{3}$ ) is envisaged for 2020. The second stage GVD-II with 14 clusters will be completed by 2023 .

\section{The Neutrino telescope Baikal-GVD}

The concept of Baikal-GVD is based on a number of evident requirements for the design and organization of the measuring system of the new detector: the utmost use of the advantages of mounting the telescope from the ice cover of Lake Baikal, the expandability of the system and ensure its effective operation already at the first stages of deployment, and the possibility of implementing different versions of arrangement and spatial density of photodetector location within the same measurement system.

The detector will utilize the deep water of Lake Baikal instrumented with optical modules (OMs) - pressure resistant glass spheres equipped with photomultiplier tubes Hamamatsu R7081100 with photocathode diameter of 10 " and a quantum efficiency of $35 \%$ [5]. The PMTs record the Cherenkov radiation from secondary particles produced in interactions of high-energy neutrinos inside or near the instrumented volume. From the arrival times of light at the PMTs and from the amount of light, direction and energy of the incoming neutrinos are derived. 

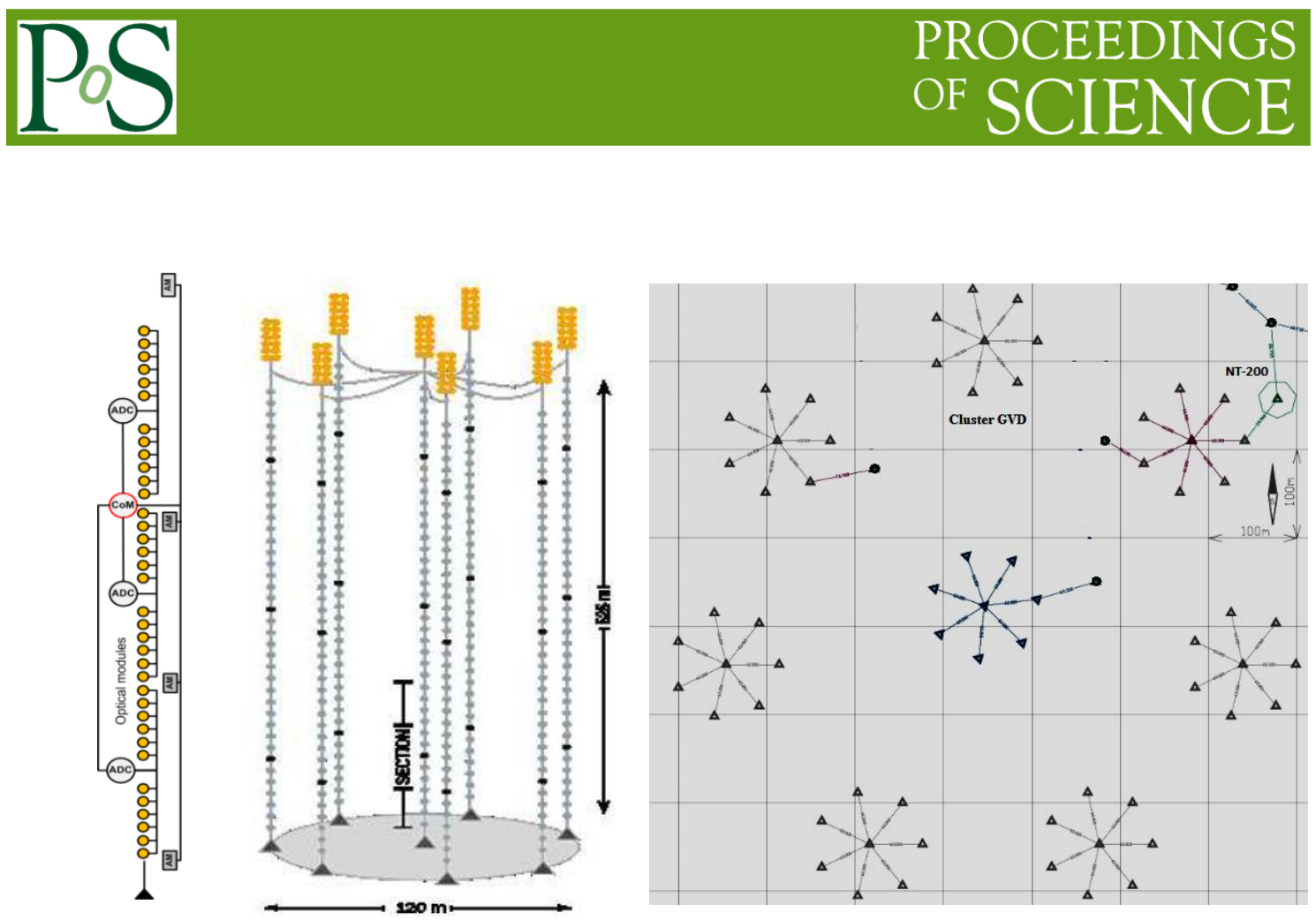

Fig. 2.1. Block diagram of the string and view of a cluster of Baikal-GVD (left), and layout of eight clusters of GVD-1 (right).

The array will consist of a network of autonomous subdetectors - so-called clusters - each of them with 288 optical modules. Each cluster is a fully functional detector which is capable of detecting a physical event both in standalone mode, and as part of a full-scale installation. A cluster comprises eight vertical strings attached to the lake floor: seven side strings on a radius of $60 \mathrm{~m}$ around a central one. Each string carries $36 \mathrm{OMs}$, arranged at depths between 735 and 1260 meters (instrumented length: $525 \mathrm{~m}$ ). The vertical spacing between the OMs along a string is 15 $\mathrm{m}$. The OMs are functionally combined in 3 sections. A section comprises $12 \mathrm{OMs}$ with data processing and communication electronics and forms a detection unit (DU) of the array. All analogue signals from the PMTs are digitized and processed in the sections and are sent to shore if certain trigger conditions (e.g. a minimum number of fired PMTs) are fulfilled. The clusters are connected to shore (3.5 km distance) via a network of cables for electrical power and highbandwidth data communication. The shore station provides power, detector control and readout, computing resources and a high-bandwidth internet connection to the data repositories. The overall design allows for a flexible and cost-effective implementation of the array. The large detection volume, combined with high angular and energy resolutions and moderate background conditions in fresh lake water allows for efficient study of cosmic neutrinos, muons from charged cosmic rays and exotic particles. It is also an attractive platform for environmental studies.

The first phase GVD-1 will comprise 8 clusters with 2304 OMs in total. The distances between the centers of the clusters are $300 \mathrm{~m}$. Figure 2.1 shows the block diagram of the string, the view of a Baikal-GVD cluster, and the layout of GVD-1. The triangles on the plan show the deep-water stations of the strings, circles indicate the cable stations installed to date. 


\section{Data acquisition system}

The basic structural unit of the data acquisition system of Baikal-GVD [6,7] is a section of OMs. A section is a functionally complete unit that includes systems of registration of radiation, calibration systems, and control electronics for the formation of trigger, the signal processing, and data transfer. Three sections of optical modules reside on the same carrying cable and form a string. The configuration of a section, which is currently the basis for the creation of the telescope, includes 12 optical modules with analog outputs, and a Central section Module (CM), which converts analog signals into a digital code. The CM is located in the middle of the section, to minimize the lengths of cables connecting it to OMs. Each measuring channel of a section consists of PMT, preamplifier and 12-bit ADC with a sampling frequency of $200 \mathrm{MHz}$ and an amplitude resolution of $1.6 \mathrm{mV}$. The conversion coefficients of the channels are leveled off at about $10^{8}$ by adjusting the high voltage of a PMT in the range from 1100 to 1800 Volts. This provides an average single-photoelectron amplitude of the channels of $\sim 25$ ADC counts.

Grouping OMs into separate sections allows to organize inter-module coincidences for suppression of the background glow of Baikal water. Coincidences of signals from any pairs of neighboring OMs with low threshold (0.5 - 1.5 p.e.) and high threshold (3 - 4 p.e.) are used as a local trigger of the section (signal request). Average rates of the section request signals are about $2-10 \mathrm{~Hz}$, in dependence of thresholds and water luminescence.

The request signals from three sections are combined in the Control Module of the string $(\mathrm{CoM})$ and transferred to the cluster $D A Q$ center, where a global trigger is formed (signal acknowledge). Signal acknowledge, returning to each section of the cluster, stops the ADCs and initiates the formation of a master record of the sections and data transmitting to the Cluster $D A Q$ center. Each master record comprises the time of the trigger, the state of the counter of the acknowledge signals (used to merge records of the same event from different sections), and waveforms for all 12 channels recorded in a time window of $5 \mu$ s (1024 ADC time counts). The full length of a master record is $12 \times 2048$ bytes. The total frequency of acknowledge signals is 50 $-250 \mathrm{~Hz}$ (the sum of the rates of requests signals of all sections of the cluster). Master records are transmitted to the Cluster DAQ center by the Ethernet network on the basis of Ethernet extenders with a transfer rate of up to $10 \mathrm{Mbit} / \mathrm{s}$. A relatively low bandwidth of network does not allow transferring master records with full record size. So, master records are converted: a part of the records are selected in which ADC data exceeds a threshold of the magnitude of $\sim 0.3$ singlephotoelectron signal. The conversion process is implemented at the hardware level. The average size of the converted master record is approximately 300 bytes for the basic mode of detector operation (muon detection). This ensures reliable transmission of the full event flow.

In addition to the electronic units directly engaged in the registration process, the data acquisition system includes a number of auxiliary subsystems: control of power supply, calibration of the detector, measuring of the coordinates of the optical modules. The system of power control allows disabling, if necessary, any of the underwater modules of the array. Managed power switches, installed in the center of the cluster, in each string and in each section, are used for these purposes. The calibration setup comprises LED light sources installed in each 
optical module (for amplitude and time calibration of the channels) and separate underwater modules with LEDs (for time calibration of the sections). The laser light source is used for the inter-cluster calibration.

The coordinates of the optical modules are determined using an acoustic positioning system. Acoustic positioning system of the cluster comprises 32 acoustic modems (AM). Four AMs are mounted on each string on the distances from of the string bottom 1, 181, 346, and 538 meters. The coordinates of the AMs are measured with an accuracy of $\sim 2 \mathrm{~cm}$. Linear interpolation is used to determine the coordinates of the optical modules located between acoustic modems.

\section{The configuration of Baikal-GVD 2017}

The modular structure of the neutrino telescope Baikal-GVD provides the ability to perform physical research in the early stages of the detector construction using different configurations of the array. The first demonstration cluster consisting of 8 strings with $24 \mathrm{OMs}$ on each (two sections) was put into operation in 2015 [8]. This array was upgraded to baseline configuration (three sections on the string) in 2016. The second cluster of the Baikal-GVD was commissioned in April 2017. Two full-scale clusters of Baikal-GVD are successfully operating during 2017. Figure 4.1 shows the present layout of the Baikal-GVD and the cumulative number of the master records for each of two clusters.
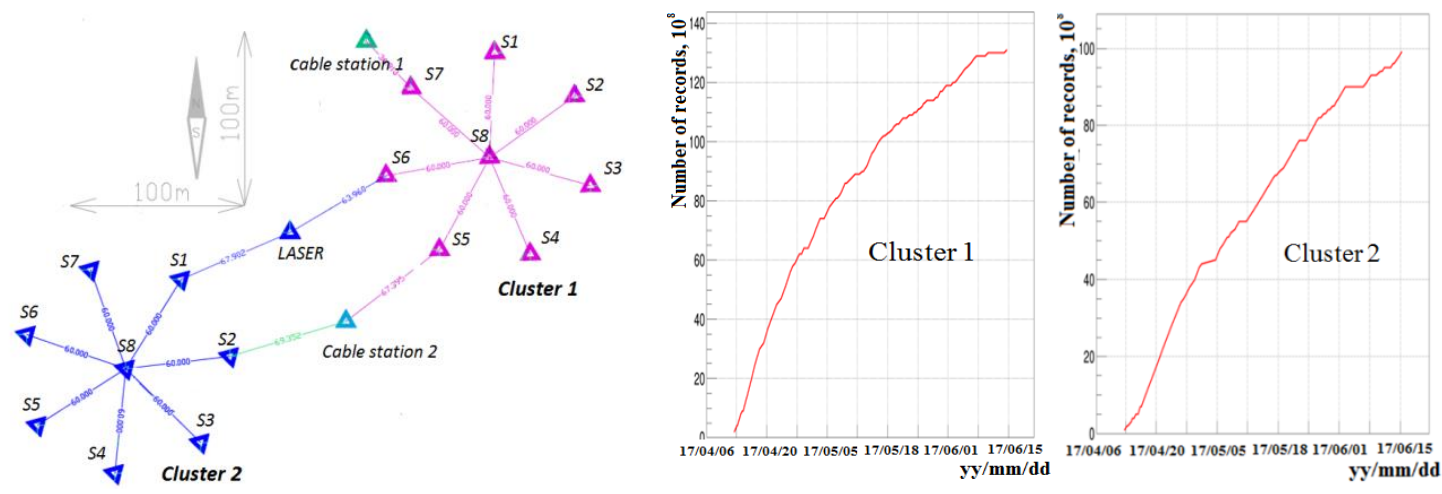

Fig. 4.1. Layout of the installation and cumulative number of the master records detected with two clusters (since April 13 to June 15 of 2017).

Each cluster consists of 8 Strings $(S 1 \ldots S 8)$ and the cluster $D A Q$ center, located on the central string (S8). The cluster DAQ centers are connected to the Shore center by hybrid optoelectrical cables providing power supply (300 VDC) and data transmission. Additional cable stations are deployed to guide the cables to the desired level from the lake bottom to $\sim 25$ meters below the lake surface and to connect them to the Cluster DAQ centers (see Fig. 4.1). The laser calibration source is mounted on a separate station (Laser string) between two clusters. Two additional acoustic modems are installed on the Laser string to measure its coordinates. 
In the period from April 13 to June 15, 2017 about $10^{7}$ events containing about $3 \times 10^{8}$ master records were detected within two months. The efficiency of the detector operation was about $85 \%$.

\section{Laser sub-nanosecond pulse light source}

The inter-string temporal calibration within a cluster is provided by dedicate LED-light sources. However, the inter-cluster calibrations require more intensive light sources. Such light source was designed and produced on a base of a powerful laser. The main $1064 \mathrm{~nm}$ light emitting element of laser is a neodymium-doped yttrium aluminum garnet (YAG:Nd3+). The beam frequency is doubled in a nonlinear crystal to a wavelength of $532 \mathrm{~nm}$. Pumping of the main element provides by laser LEDs with a wavelength of $808 \mathrm{~nm}$. The pulse energy reaches a value of $0.37 \mathrm{~mJ}\left(\sim 10^{15}\right.$ photons), the flash duration is about $1 \mathrm{~ns}$. A light diffuser is installed at the output of the laser beam providing an isotropic light emission in water. Special sensor is used for control of output light intensity. An adjustment of the flush intensity is provided by light attenuation system. Software stuffing allows for operation in different modes, changing the number, frequency and power of light flashes during flashing session. The laser is installed at about the same depth as the center of the lower sections. The photograph of the laser light source is shown in Fig. 5.1 (left).
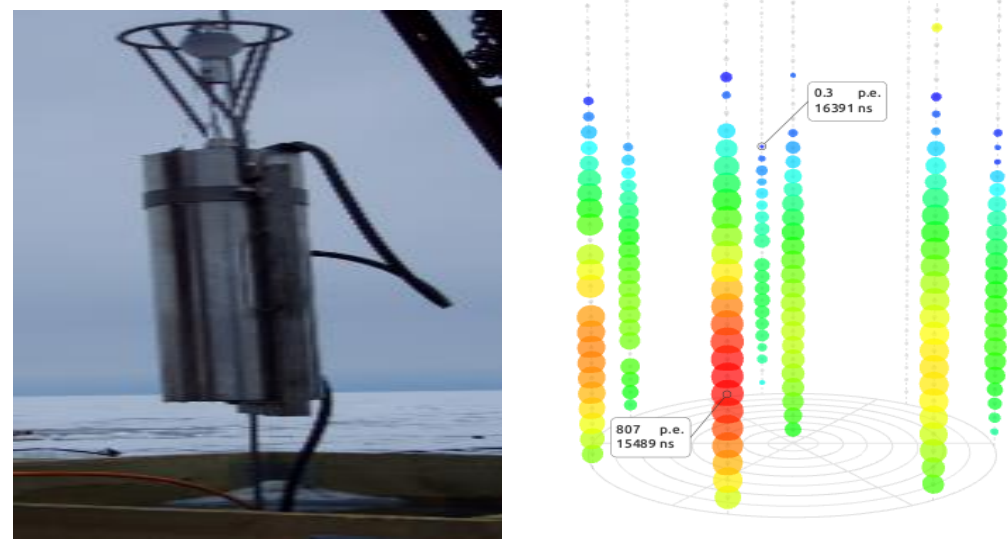

Fig.5.1 The photograph of laser light sources before deployment (left). Laser event detected by GVD cluster (right).

An example of a laser event detected with Cluster-1 is presented in Fig. 5.1 (right), where the sphere size is proportional to the light intensity and the color indicates the light arrival time at the OM. The amplitudes and light arrival times recorded by OMs are shown in Fig. 5.2. The numbering of the channels starts with the bottom OM of the first string: from 1 to 288 . The lack of data on the second section is due to the fact that two sections were excluded from the configuration. Laser flashes are reliably detected by all channels of the lower and middle sections of the strings. Time calibration of the channels was provided by using LED calibration sources of the cluster. The solid lines in Fig. 5.2 (right) show the expected times of the light propagation 
from the laser to the OMs, which were calculated using the information about OMs and laser positions that have been provided by the acoustic positioning system. The good agreement between measured and expected times of registration of the laser flashes (see Fig. 5.2) indicates the correctness of the channel calibration and a sufficiently high positioning accuracy of the optical modules measured by the acoustic system.
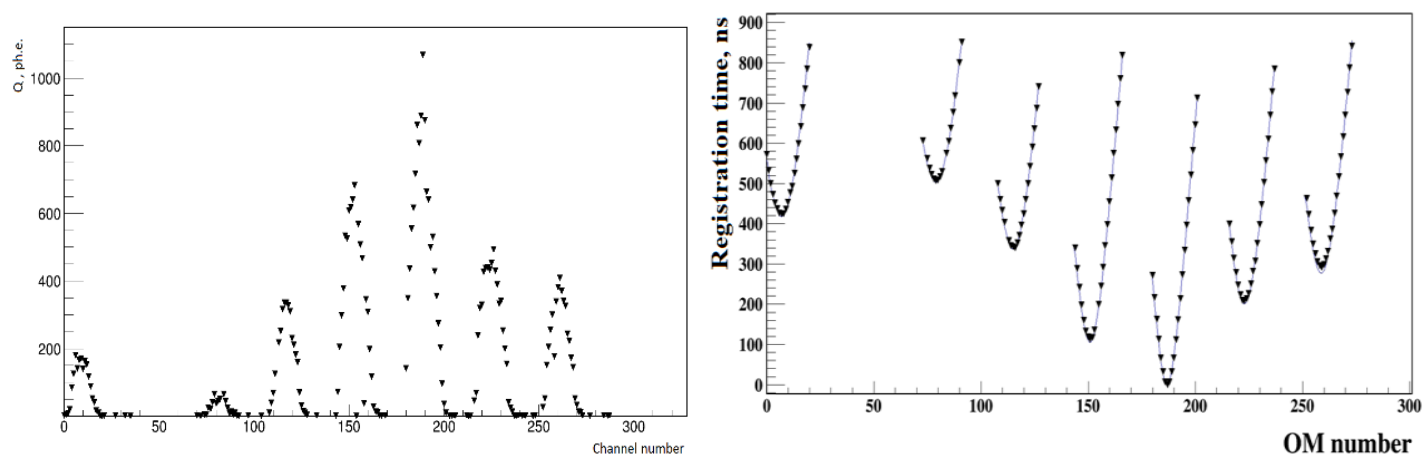

Fig.5.2 Amplitude (left) and time (right) distributions of signals recorded by OMs for laser event.

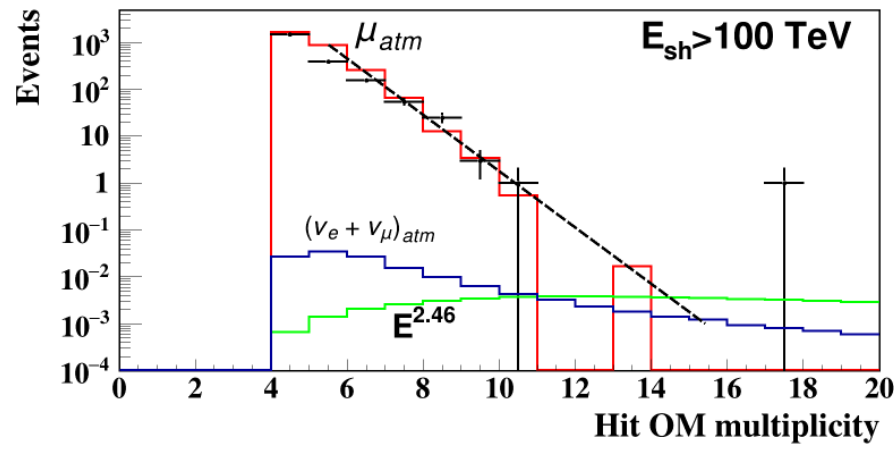

Fig. 5.1. The multiplicity distribution of hit channels for cascade-like events with energies above $100 \mathrm{TeV}$; points - experiment, red line - background of atmospheric muons, blue line - background of atmospheric neutrinos, green line - expected signal from astrophysical neutrinos.

\section{Cascade shower detection}

The registration of neutrinos with Baikal-GVD is carried out by detecting the products of their interactions which appear as muon tracks and cascade showers. A data sample obtained with the first cluster of Baikal-GVD in the period 24 Oct to 17 Dec 2015 was used for investigating and checking the methods of the selection from background events and reconstruction of the cascade showers (41.6 days of the detector live time). About $4.4 \times 10^{8}$ triggered events were detected by the array during this time period. After applying several selection cuts and reconstruction of cascades vertex, energy and direction, 1192 events with reconstructed energies $\mathrm{E}>100 \mathrm{TeV}$ have been selected. Fig. 6.1 shows the distribution of these events with respect to the multiplicity of hit channels. The experimental distribution is consistent with the expectation from atmospheric muons for multiplicities $N_{h i t}<10$. The expected $N_{h i t}$ distributions for events 
induced by atmospheric neutrinos and neutrinos of astrophysical nature (IceCube $E^{-2.46}$ neutrino spectrum) are also shown in Fig. 6.1. One eye-catching event with $N_{h i t}=17$ was found in the selected data sample. This event was reconstructed as high-energy cascade with an energy of 107 $\mathrm{TeV}$ and a zenith angle of $56.6^{\circ}$. The expected probability of an event with $\mathrm{N}_{\text {hit }}>16$ is about $5 \%$ for astrophysical neutrino flux, and less than $1 \%$ for atmospheric neutrinos. More MC-simulation statistics is required for a precise estimation of the expected probability of such events for atmospheric muons.

\section{Conclusion}

The ambition of the Baikal collaboration is to construct a $\mathrm{km}^{3}$-scale neutrino telescope: the Gigaton Volume Detector in Lake Baikal, with implementation of about ten thousand photodetectors. The stage of prototyping of the GVD project has been performed since April 2011 and aimed at comprehensive in situ tests of all elements and systems of the future detector. This phase was concluded in 2015 with the deployment and operation of the first demonstration cluster "Dubna". In 2016 this array was upgraded to the baseline configuration of a GVD cluster with 288 OMs arranged on eight vertical strings. The second full-scale GVD cluster was installed and commissioned in April 2017. The operation of both clusters has been successfully tested with the use of a new laser calibration source that was deployed in 2017. The effective volume of a present GVD configuration for high energy cascades is about $0.1 \mathrm{~km}^{3}$. The commissioning of the first stage of the Baikal neutrino telescope GVD-1 with an effective volume $0.4 \mathrm{~km}^{3}$ is envisaged for 2020.

The Baikal-GVD project is supported by the RFBR grants 16-29-13032, 17-02-01237.

\section{References}

[1] A.D. Avrorin et al., Baikal-GVD, EPJ Web Conf. 136, 04007 (2017).

[2] K. Antipin et al., Physics Results from the Baikal Neutrino Telescope, Nuclear Physics B (Proc. Suppl.) 168 (2007) 296-298.

[3] V. Aynutdinov, et al., The prototype string for the km3-scale Baikal neutrino telescope, NIM A602 (2009) 227-234.

[4] V. Aynutdinov et al., The prototyping/early construction phase of the BAIKAL-GVD project, NIM A742 (2014) 82-88.

[5] A.D. Avrorin et al., The optical module of Baikal-GVD, EPJ Web Conf. 116, 01003 (2016).

[6] A.V. Avrorin et. Al., Status of the Baikal-GVD project, NIM A 692 (2012) 46-52.

[7] V. Aynutdinov et al., The data acquisition system for Baikal-GVD, EPJ Web of Conf. 116, 05004(2016).

[8] A.D. Avrorin et al., Status of the early construction phase of Baikal-GVD, Nuclear and Particle Physics Proceedings 273-275 (2016) 314-320. 